\title{
Lung transplantation in patients 70 years old or older: Have outcomes changed after implementation of the lung allocation score?
}

\author{
Arman Kilic, MD, ${ }^{a}$ Christian A. Merlo, MD, MPH, ${ }^{\mathrm{b}}$ John V. Conte, MD, ${ }^{\mathrm{a}}$ and Ashish S. Shah, $\mathrm{MD}^{\mathrm{a}}$
}

Objective: The objective of the present study was to evaluate whether the outcomes of lung transplantation in patients aged 70 years or older have changed after implementation of the lung allocation score in May 2005.

\begin{abstract}
Methods: Patients aged 70 years or older undergoing primary lung transplantation from 1995 to 2009 were identified from the United Network for Organ Sharing registry. The primary stratification was the pre-lung allocation score era versus lung allocation score era. Risk-adjusted multivariate Cox regression and Kaplan-Meier analyses were conducted to evaluate the effect of age 70 years or older on 1-year post-transplant mortality compared with a reference cohort of patients aged 60 to 69 years.
\end{abstract}

Results: Of the overall 15,726 adult lung transplantation patients in the study period, $225(1.4 \%)$ were 70 years old or older and $4634(29.5 \%)$ were 60 to 69 years old. The patients aged 70 years or older were a larger cohort of overall lung transplantation patients in the lung allocation score era compared with before the lung allocation score era $(3.1 \%$ vs $0.3 \%, P<.001)$. In the risk-adjusted Cox analysis, age 70 years or older was a significant risk factor for 1-year post-lung transplantation mortality in the pre-lung allocation score era (hazard ratio, 2.00; $95 \%$ confidence interval, 1.10-3.62, $P=.02$ ) but not in the lung allocation score era (hazard ratio, 1.02; 95\% confidence interval, $0.71-1.46 ; P=.92)$. Similarly, Kaplan-Meier 1 -year survival was significantly reduced in patients 70 years old or older versus 60 to 69 years old in the pre-lung allocation score era $(56.7 \%$ vs $76.3 \%$, $P=.006)$ but not in the lung allocation score era $(79.0 \%$ vs $80.0 \%, P=.72)$.

Conclusions: Recipients aged 70 years or older were a larger proportion of overall lung transplantation patients after implementation of the lung allocation score. Although associated with significantly increased post-lung transplantation mortality in the pre-lung allocation score era, age 70 years or older is currently associated with outcomes comparable to those of patients aged 60 to 69 years. Therefore, age 70 years or older should not serve as an absolute contraindication to lung transplantation in the lung allocation score era. ( $\mathrm{J}$ Thorac Cardiovasc Surg 2012;144:1133-8)

With an aging population in the United States, the number of patients with end-stage lung disease will undoubtedly continue to increase. This again brings into question the long debated issue of what the upper age limit should be for recipients of lung transplantation (LTx). A consensus report published in 2006 from the International Society for

From the Division of Cardiac Surgery, ${ }^{a}$ Department of Surgery, and Division of Pulmonary and Critical Care Medicine, ${ }^{\mathrm{b}}$ Department of Medicine, Johns Hopkins Medical Institutions, Baltimore, Md.

Supported by departmental funds from the Department of Surgery, Johns Hopkins Hospital, Baltimore, Md, and, in part, by Health Resources and Services Administration contract 234-2005-37011C.

Disclosures: Authors have nothing to disclose with regard to commercial support.

The content is the responsibility of the authors alone and does not necessarily reflect the views or policies of the Department of Health and Human Services, nor does mention of trade names, commercial products, or organizations imply endorsement by the US Government.

Read at the 92nd Annual Meeting of The American Association for Thoracic Surgery, San Francisco, California, April 28-May 2, 2012.

Received for publication April 1, 2012; revisions received July 11, 2012; accepted for publication July 30, 2012; available ahead of print Sept 3, 2012.

Address for reprints: Ashish S. Shah, MD, Division of Cardiac Surgery, Department of Surgery, Johns Hopkins Hospital, 600 North Wolfe St, Blalock 618, Baltimore, MD 21287 (E-mail: ashah29@jhmi.edu).

$0022-5223 / \$ 36.00$

Copyright (c) 2012 by The American Association for Thoracic Surgery

http://dx.doi.org/10.1016/j.jtcvs.2012.07.080
Heart and Lung Transplantation recommended that recipient age older than 65 years should serve as a relative contraindication to LTx. ${ }^{1}$ This recommendation was based on International Society for Heart and Lung Transplantation registry data demonstrating reduced survival in this elderly cohort. $^{2}$ It is unknown whether the implementation of the lung allocation score (LAS) in May 2005 has been met with any changes in outcomes after LTx in elderly recipients. In the present study, we evaluated the trends and outcomes of LTx in recipients aged 70 years or older in the United States.

\section{PATIENTS AND METHODS \\ Data Source}

The United Network for Organ Sharing (UNOS) data set was used for the present study. The UNOS registry collects patient-level data on all transplantations performed in the United States. The Johns Hopkins University School of Medicine approved our study.

\section{Study Population}

All adult patients (age $>17$ years) undergoing LTx between January 1, 1995, and December 31, 2009 were initially identified in the UNOS data set. Patients undergoing multivisceral transplantation, including heartlung transplantation, were excluded. Redo lung transplants were also excluded from the analysis. 


\section{Abbreviations and Acronyms \\ LAS = lung allocation score \\ LTx = lung transplantation \\ $\mathrm{UNOS}=$ United Network for Organ Sharing}

\section{Trends in LTx in Elderly Recipients}

To examine nationwide trends, we stratified the recipients into 3 age groups: 18 to 59 years, 60 to 69 years, and 70 years old or older. The absolute number of recipients within each of these age cohorts was then plotted for each year during the study period. The trends were then evaluated by calculating the correlation coefficients $(r)$ and the associated $P$ values.

We also compared the trends in key baseline characteristics of elderly recipients. Moreover, the recipient, donor, and transplant variables for recipients aged 70 years or older were compared between those who underwent transplantation in the pre-LAS era versus the LAS era. These included the following recipient variables: age, gender, race, weight, height, body mass index, etiology of lung disease, recent infection, dialysis while on the wait list, serum creatinine, serum bilirubin, serum cytomegalovirus positivity, bridging with extracorporeal membrane oxygenation, mechanical ventilation before transplantation, intensive care unit before transplantation, diabetes mellitus, hypertension, previous malignancy, and blood transfusion while on the wait list. Donor variables that were compared included age, gender, race, weight, height, body mass index, serum cytomegalovirus positivity, cigarette use, diabetes mellitus, terminal creatinine, hypertension, inotrope use, and mechanism of death. The transplant variables included transplant type (single versus bilateral LTx), days on the wait list, ischemic time, and center volume. Pairing data between the recipient and donor included gender matching, race matching, blood type matching, donor/recipient body mass index ratio, donor/recipient weight ratio, donor/recipient height ratio, human leukocyte antigen matching, and cytomegalovirus status matching.

\section{Risk-Adjusted Cox Regression Analysis}

Multivariate Cox regression models were constructed to examine the risk-adjusted effect of recipient age 70 years or older on 1-year all-cause mortality after LTx. The reference cohorts in these analyses were recipients aged 60 to 69 years. Risk adjustment was performed for the recipient, donor, and transplant variables that were associated with 1-year mortality on exploratory univariate analysis $(P<.2)$ and had less than $20 \%$ missing data. All the variables listed previously were tested in this exploratory analysis for potential inclusion in the multivariate models. Significant interactions between covariates were thoroughly tested.

\section{Kaplan-Meier Survival Analysis}

Kaplan-Meier survival curves were also constructed. These curves were stratified according to age cohort (age 60-69 years vs age $\geq 70$ years) and era (pre-LAS era vs LAS era). The log-rank test was used to compare the survival curves between the age groups within each era.

\section{Statistical Analysis}

The categorical data are presented as the number and percentage and were compared using the chi-square test or Fisher's exact test. Continuous data are presented as the mean \pm standard deviation and were compared using the Student $t$ test. All statistical analyses were performed using STATA, software version 11 (StataCorp, College Station, Tex).

\section{RESULTS \\ Study Cohort}

A total of 16,823 adult patients underwent LTx from 1995 to 2009 in the UNOS data set. Of these, 562 were re-do LTx, 495 were heart-lung transplants, and 40 were other types of multivisceral transplants. After excluding these cases, 15,726 adult first-time, single-organ LTx patients were included in the study. Of these, $225(1.4 \%)$ were 70 years old or older and $4634(29.5 \%)$ were $60-69$ years old.

\section{Trends in LTx in Elderly Recipients}

Several significant trends were observed with regard to recipient age during the study period. The proportion of adult LTx recipients aged 18 to 59 years decreased significantly from $85.0 \%$ in 1995 to $52.5 \%$ in $2009(P<.001$; Figure 1). During the same period, the proportion of recipients aged 60 to 69 years increased from $14.8 \%$ to $43.0 \%$ and the proportion of recipients aged 70 years or older increased from $0.3 \%$ to $4.5 \%(P<.001)$. When stratified according to the implementation of the LAS, the proportion of LTx recipients who were 70 years or older increased from $0.3 \%(\mathrm{n}=30$ of 9,338) in the pre-LAS era to $3.1 \%(\mathrm{n}=195$ of 6391$)$ in the LAS era $(P<.001)$.

\section{Baseline Characteristics}

Also, significant trends were seen when comparing the baseline characteristics of recipients aged 70 years or older in the pre-LAS versus the LAS era. Moreover, septuagenarian recipients in the LAS era were older (Table 1). The mean serum creatinine level in the LAS group was significantly lower than in the pre-LAS cohort, although no patients in either septuagenarian cohort underwent dialysis while on the wait list. Similar to previous data examining the effect of the LAS for all LTx patients, elderly LTx patients in our study in the LAS era had shorter wait list times and a greater proportion were patients with idiopathic pulmonary fibrosis with fewer having chronic obstructive pulmonary disease. The mean LAS in the septuagenarian LTx recipients in the modern cohort was $47.2 \pm 16.0$.

The donors for septuagenarian recipients were wellmatched between eras (Table 2). The recipient-donor pairing data were also comparable. Centers performing LTx in recipients aged 70 years or older had significantly greater volumes in the LAS era than in the pre-LAS era, performing on average 9 more LTx annually.

\section{Risk-Adjusted Cox Regression Analysis}

On univariate Cox regression analysis, recipients aged 70 years or older were more than twice as likely to die within 1 year of LTx as recipients aged 60 to 69 years in the pre-LAS era (hazard ratio, 2.13; $95 \%$ confidence interval, $1.23-3.70 ; P=.007)$. In the LAS era, age 70 years or older was not a significant risk factor for 1-year mortality on univariate analysis (hazard ratio, 1.06; 95\% confidence interval, $0.77-1.47 ; P=.72$ ). Similar trends were observed after risk adjustment for significant recipient, donor, and transplant variables in the pre-LAS versus LAS eras. 


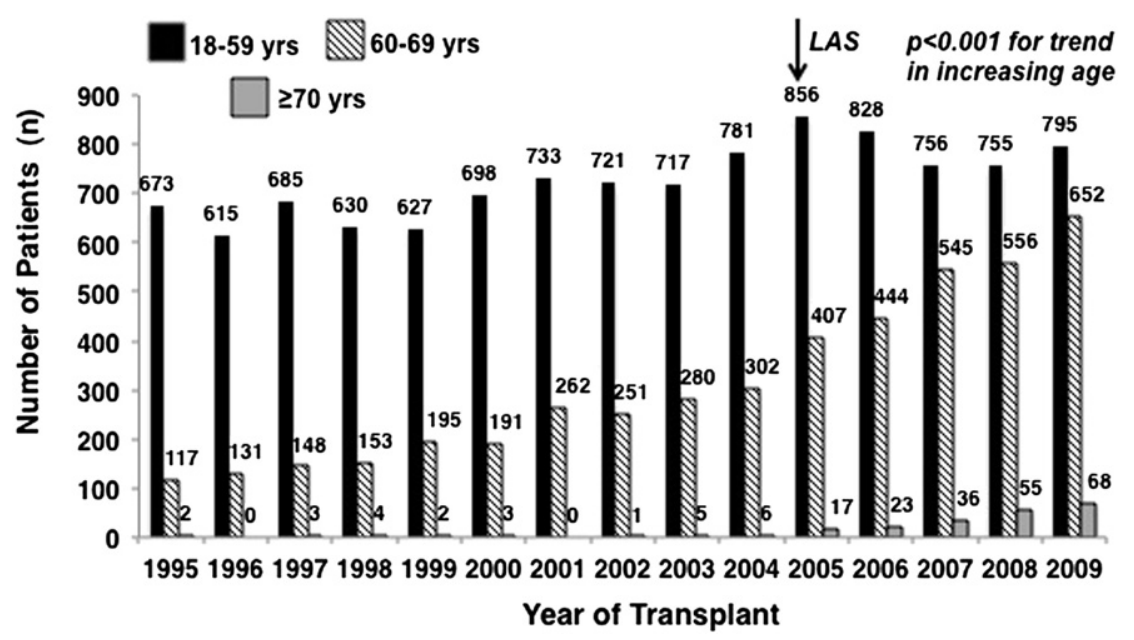

FIGURE 1. Trends in lung transplantation ( $L T x)$ recipient age during the study period.

Moreover, in the pre-LAS era, the risk-adjusted hazard ratio for 1-year mortality in those aged 70 years or older compared with those aged 60 to 69 years was $2.00(95 \%$ confidence interval, 1.10-3.62), a statistically significant difference $(P=.02$; Table 3$)$. Conversely, age 70 years or older did not exert an independent effect on 1-year mortality in risk-adjusted analysis in the LAS era (hazard ratio, $1.02 ; 95 \%$ confidence interval, 0.71-1.46; $P=.92$ ).

\section{Kaplan-Meier Survival Analysis}

Kaplan-Meier survival analysis revealed the same trends as observed in the Cox regression analysis. In the pre-LAS era, the 1-year survival for patients aged 60 to 69 years was $76.3 \%$, significantly greater than the $56.7 \%$ observed for patients aged 70 years or older (log-rank $P=.006$; Figure 2). After implementation of the LAS, however, the 1 -year survival for recipients aged 60 to 69 years versus

TABLE 1. Baseline recipient characteristics in recipients aged 70 years or older, stratified by era

\begin{tabular}{|c|c|c|c|}
\hline Recipient characteristic & $\begin{array}{l}\text { Before LAS } \\
\text { era }(n=30)\end{array}$ & $\begin{array}{c}\text { LAS } \\
\text { era }(n=195)\end{array}$ & $\begin{array}{c}P \\
\text { value }\end{array}$ \\
\hline Age (y) & $71.1 \pm 1.6$ & $72.0 \pm 2.2$ & $<.001$ \\
\hline Female gender & $5 / 30(16.7 \%)$ & $39 / 195(20.0 \%)$ & .67 \\
\hline White race & $29 / 30(96.7 \%)$ & $179 / 195(92.3 \%)$ & .38 \\
\hline Diagnosis & & & .04 \\
\hline IPF & $18 / 30(60.0 \%)$ & $145 / 195(74.4 \%)$ & \\
\hline COPD & $12 / 30(40.0 \%)$ & $40 / 195(20.5 \%)$ & \\
\hline Other & $0 / 30(0.0 \%)$ & $10 / 195(5.1 \%)$ & \\
\hline Bridging with ECMO & $0 / 30(0.0 \%)$ & $2 / 193(1.0 \%)$ & .58 \\
\hline Mechanical ventilation & $0 / 30(0.0 \%)$ & $14 / 195(7.2 \%)$ & .13 \\
\hline $\begin{array}{l}\text { Serum cytomegalovirus } \\
\text { positive }\end{array}$ & $15 / 30(50.0 \%)$ & $120 / 195(61.5 \%)$ & .23 \\
\hline Serum creatinine $(\mathrm{mg} / \mathrm{dL})$ & $1.03 \pm 0.37$ & $0.91 \pm 0.23$ & .01 \\
\hline
\end{tabular}

recipients aged 70 years or older was comparable $(80.0 \%$ vs $79.0 \%$; log-rank $P=.72$ ).

\section{DISCUSSION}

In the present study, we reviewed the trends and outcomes of LTx in 225 recipients aged 70 years or older. We found that the proportion of LTx recipients who are elderly has increased significantly during the past 15 years in the United States. Also, several key changes have occurred in the characteristics of septuagenarian patients undergoing LTx with the introduction of the LAS. These include an

TABLE 2. Baseline donor, transplant, and recipient-donor matching characteristics in recipients aged 70 years or older, stratified by era

\begin{tabular}{lccc}
\hline \multicolumn{1}{c}{ Characteristic } & $\begin{array}{c}\text { Pre-LAS } \\
\text { era }(\mathbf{n}=\mathbf{3 0})\end{array}$ & $\begin{array}{c}\text { LAS } \\
\text { era }(\mathbf{n}=\mathbf{1 9 5})\end{array}$ & $\begin{array}{c}\boldsymbol{P} \\
\text { value }\end{array}$ \\
\hline Donor & & & \\
Age (y) & $36.2 \pm 15.5$ & $35.1 \pm 14.9$ & .73 \\
Female gender & $5 / 30(16.7 \%)$ & $39 / 195(20.0 \%)$ & .67 \\
White race & $21 / 30(70.0 \%)$ & $105 / 195(53.9 \%)$ & .10 \\
Mechanism of death & & & .42 \\
Trauma & $17 / 30(56.7 \%)$ & $86 / 195(44.1 \%)$ & \\
Cerebrovascular & $10 / 30(33.3 \%)$ & $79 / 195(40.5 \%)$ & \\
Other & $3 / 30(10.0 \%)$ & $30 / 195(15.4 \%)$ & \\
Serum cytomegalovirus & $17 / 30(56.7 \%)$ & $126 / 195(64.6 \%)$ & .40 \\
positive & & & \\
LTx & & & \\
Bilateral LTx & $3 / 30(10.0 \%)$ & $43 / 195(22.1 \%)$ & .13 \\
Wait list duration (d) & $165.1 \pm 237.9$ & $76.3 \pm 128.5$ & .002 \\
Annual center volume & $24.5 \pm 15.6$ & $33.6 \pm 19.8$ & .02 \\
Ischemic time (h) & $4.3 \pm 1.9$ & $4.6 \pm 1.4$ & .48 \\
Recipient-donor matching & & & \\
Gender matched & $22 / 30(73.3 \%)$ & $140 / 195(71.8 \%)$ & .86 \\
Race matched & $20 / 30(66.7 \%)$ & $107 / 195(54.9 \%)$ & .23 \\
Cytomegalovirus matched & $16 / 30(53.3 \%)$ & $103 / 195(52.8 \%)$ & .96 \\
Donor/recipient BMI ratio & $1.04 \pm 0.21$ & $1.00 \pm 0.27$ & .49 \\
\hline LAS, Lung allocation score; $L T x$, lung transplantation; $B M I$, body mass index.
\end{tabular}


TABLE 3. Risk-adjusted multivariate analysis for 1-year mortality

\begin{tabular}{lcc}
\hline Recipient age $(\mathbf{y})$ & 1-y Mortality & $\boldsymbol{P}$ value \\
\hline $60-69$ & & - \\
Pre-LAS era* & Reference & \\
LAS era $\dagger$ & Reference & \\
$\geq 70$ & & .02 \\
Pre-LAS era* & $2.00(1.10-3.62)$ & .92 \\
LAS era $\dagger$ & $1.02(0.71-1.46)$ & \\
\hline
\end{tabular}

Data presented as hazard ratio ( $95 \%$ confidence interval). $L A S$, Lung allocation score; $L T x$, lung transplantation; $I C U$, intensive care unit; $E C M O$, extracorporeal membrane oxygenation. *Risk-adjusted for significant predictors of mortality in pre-LAS era: serum creatinine, serum bilirubin, year of transplantation, wait list duration, race, mechanical ventilation before LTx, diabetes mellitus, ICU before LTx, etiology of lung disease, serum cytomegalovirus status, donor weight, donor height, donor hypertension, recipient-donor gender matching, and center volume. $\dagger$ Risk-adjusted for significant predictors of mortality in LAS era: serum creatinine, serum bilirubin, year of transplantation, wait list duration, race, gender, mechanical ventilation before LTx, ICU prior to LTx, bridging with ECMO, lung allocation score, recent infection, serum cytomegalovirus status, dialysis while on wait list, blood transfusion while on wait list, donor height, donor cytomegalovirus status, donor cigarette use, donor diabetes, cytomegalovirus status match, and center volume.

older elderly cohort in the LAS era and changes that have been appreciated in the general LTx population such as a greater percentage of patients with idiopathic pulmonary fibrosis, a lower percentage of recipients with chronic obstructive pulmonary disease, and shorter wait list times. ${ }^{3,4}$

In addition to these changes, the rate of bilateral LTx in septuagenarians was more than double in the modern cohort than in the pre-LAS group, although this did not reach statistical significance. Several groups have demonstrated improved survival in patients undergoing bilateral LTx compared with single LTx for a variety of indications. ${ }^{5,6}$ This benefit seems less clear in older recipients, with studies demonstrating conflicting results. ${ }^{7-10}$ Regardless, it does appear from our analysis that more groups are willing to perform bilateral LTx in elderly patients, although the vast majority continue to be single LTx.

The mean LAS in the modern cohort of septuagenarians in our study was 47.2 , with $38 \%$ (74/195) of these patients having an LAS greater than 46 , corresponding to the greatest quintile of risk in the general adult LTx population. ${ }^{11}$ Therefore, the 1-year survival of $79.0 \%$ demonstrated in the present cohort in the LAS era was acceptable. Furthermore, although septuagenarians had increased risk-adjusted mortality compared with a control cohort of recipients aged 60 to 69 years in the pre-LAS era, we found no significant increase in 1-year mortality in septuagenarians undergoing LTx after implementation of the LAS.

\section{Implications}

The improvements in outcomes in septuagenarians undergoing LTx are likely a reflection of clinical improvements in transplant technique, postoperative care, and immunosuppression, among other factors. It is unclear from our analysis to what degree the implementation of the LAS system directly attributed to these improvements. Nonetheless, it does appear from our data that outcomes have substantially improved in recent years in this elderly cohort of recipients. This has occurred despite more patients with idiopathic pulmonary fibrosis, which represent a higher risk subset and for which LTx offers a more limited survival benefit than for other indications. ${ }^{12}$ Therefore, the collective data presented in our analysis are encouraging and suggest that advanced age alone should not serve as an absolute contraindication to LTx.

Although we adjusted for baseline differences between groups, it is possible that improvements in patient selection in the elderly cohort could have contributed to the better survival observed in the recent era. One favorable

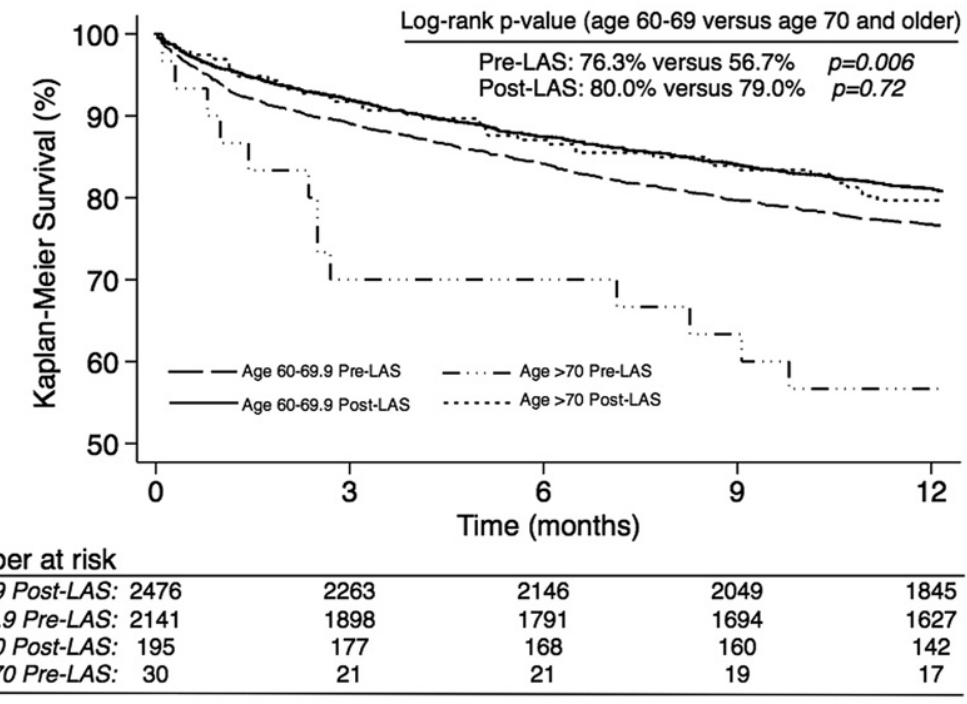

FIGURE 2. Kaplan-Meier survival curves stratified by recipient age and era. LAS, Lung allocation score. 
factor in the recent septuagenarian cohort was a lower serum creatinine compared with septuagenarians transplanted in the earlier era. Preoperative creatinine is a known risk factor for developing renal failure requiring dialysis postoperatively, a complication associated with significant increases in mortality. A study of more than 12,000 LTx recipients confirmed the importance of preoperative renal function in determining postoperative renal failure risk. Furthermore, they demonstrated a more than sevenfold increase in 30-day and 1-year mortality and more than fivefold increase in 5-year mortality in those developing renal failure after LTx compared with those who did not. ${ }^{13}$

Another favorable factor noted in the more recent septuagenarian cohort was greater center volume. A study of 10,000 adult recipients found that centers with higher volumes had the lowest short-term mortality rates after LTx. ${ }^{14}$ Another nationwide analysis confirmed this association between increasing center volume and decreasing short-term mortality. ${ }^{15}$ Furthermore, it demonstrated that this relationship has become more pronounced in recent years. ${ }^{15}$ It is unclear from our analysis whether LTx in the older population has been consolidated to high-volume centers in recent years or whether the higher center volume is merely a reflection of more LTx procedures being performed over time.

\section{Previous Studies}

Several previous studies have evaluated the effect of older recipient age on outcomes after LTx. A singlecenter review of 52 LTx patients aged 60 to 69 years demonstrated comparable short- and long-term survival compared with younger recipients. ${ }^{16}$ Another institutional series of 32 LTx recipients aged 60 years or older also demonstrated comparable survival, complication rates, graft function, and frequency of bronchiolitis obliterans syndrome compared with patients younger than 60 years old. ${ }^{17}$ Similarly, a previous multicenter study by our group focusing on predominantly pre-LAS patients found that age older than 60 years was associated with comparable 30-day mortality compared with younger patients. ${ }^{18}$ Septuagenarians were at high risk of early mortality in that previous analysis, similar to the pre-LAS cohort in our present study, although our present study population added an additional 168 septuagenarian recipients in the LAS era and demonstrated significantly improved survival over time.

\section{Study Limitations}

Similar to other multicenter registries, the UNOS data set is susceptible to missing data entries and inaccurately entered data. The registry is also limited to transplants performed by centers within the United States. Although some comorbidity data are available in the UNOS registry, a validated index such as the Charlson or Deyo could not be calculated for the present study but could be an important measure, especially when discussing the physiologic versus chronologic patient age. In addition, we limited our analysis to the primary outcome of all-cause mortality, although other outcomes, including quality of life, functional capabilities, and resource usage are also important measures, particularly in this patient cohort. We also did not include long-term survival as an outcome measure, primarily because the LAS cohort did not have sufficient follow-up to conduct such analyses. In addition, it is unclear from our analysis whether the implementation of the LAS directly affected outcomes in elderly patients or whether these merely reflect ongoing changes over time. Finally, the present study was susceptible to type II error, although a significant statistical difference was observed in the smaller pre-LAS cohort, which was most susceptible to this error. Furthermore, in the larger LAS era cohort, the absolute difference in 1-year survival versus controls was very small $(1.0 \%)$, suggesting that the outcomes were likely comparable in actuality and not just a limitation of sample size.

\section{CONCLUSIONS}

The present study is the largest to date examining outcomes of LTx in septuagenarians. In this review of 225 LTx recipients aged 70 years or older, survival was comparable to that of patients aged 60 to 69 years in the LAS era, with significant improvements relative to pre-LAS septuagenarians. These data have collectively demonstrated that with appropriate patient selection, favorable posttransplant outcomes can be achieved in elderly recipients. Therefore, advanced age should not serve as an absolute contraindication to LTx in the present era.

\section{References}

1. Orens JB, Estenne M, Arcasoy S, Conte JV, Corris P, Egan JJ, et al. International guidelines for the selection of lung transplant candidates: 2006 updatea consensus report from the Pulmonary Scientific Council of the International Society for Heart and Lung Transplantation. J Heart Lung Transplant. 2006 25:745-55.

2. Trulock EP, Edwards LB, Taylor DO, Boucek MM, Keck BM, Hertz MI. Registry of the International Society for Heart and Lung Transplantation twenty-second official adult lung and heart-lung transplant report-2005. J Heart Lung Transplant. 2005;24:956-67.

3. Yusen RD, Shearon TH, Qian Y, Kotloff R, Barr ML, Sweet S, et al. Lung transplantation in the United States, 1998-2008. Am J Transplant. 2010;10: 1047-68.

4. Carr G, Garrity ER Jr. Lung transplantation outcome changes from the new US lung allocation system. Clin Pulm Med. 2012;19:39-43.

5. Thabut G, Christie JD, Ravaud P, Castier Y, Brugiere O, Fournier M, et al. Survival after bilateral versus single lung transplantation for patients with chronic obstructive pulmonary disease: a retrospective analysis of registry data. Lancet. 2008;371:744-51.

6. Weiss ES, Allen JG, Merlo CA, Conte JV, Shah AS. Survival after single versus bilateral lung transplantation for high-risk patients with pulmonary fibrosis. Ann Thorac Surg. 2009;88:1616-25.

7. Meyer DM, Bennett LE, Novick RJ, Hosenpud JD. Single vs bilateral, sequential lung transplantation for end-stage emphysema: influence of recipient 
age on survival and secondary end-points. J Heart Lung Transplant. 2001;20: 935-41.

8. Mahidhara R, Bastani S, Ross DJ, Saggar R, Lynch J III, Schnickel GT, et al. Lung transplantation in older patients? J Thorac Cardiovasc Surg. 2008;135: 412-20.

9. Fischer S, Meyer K, Tessmann R, Meyer A, Gohrbandt B, Simon A, et al. Outcome following single vs bilateral lung transplantation in recipients 60 years of age and older. Transplant Proc. 2005;37:1369-70.

10. Palmer SM, Davis RD, Simsir SA, Lin SS, Hartwig M, Reidy MF, et al. Successful bilateral lung transplant outcomes in recipients 61 years of age and older. Transplantation. 2006;81:862-5.

11. Merlo CA, Weiss ES, Orens JB, Borja MC, Diener-West M, Conte JV, et al. Impact of U.S. lung allocation score on survival after lung transplantation. J Heart Lung Transplant. 2009;28:769-75.

12. Christie JD, Edwards LB, Kucheryavaya AY, Benden C, Dobbels F, Kirk R, et al. The registry of the International Society for Heart and Lung Transplantation: twenty-eighth adult lung and heart-lung transplant report-2011. J Heart Lung Transplant. 2011;30:1104-22.
13. George TJ, Arnaoutakis GJ, Beaty CA, Pipeling MR, Merlo CA, Conte JV, et al. Acute kidney injury increases mortality after lung transplantation. Ann Thorac Surg. 2012;94:185-92.

14. Weiss ES, Allen JG, Meguid RA, Patel ND, Merlo CA, Orens JB, et al. The impact of center volume on survival in lung transplantation: an analysis of more than 10,000 cases. Ann Thorac Surg. 2009;88:1062-70.

15. Scarborough JE, Bennett KM, Davis RD, Lin SS, Tracy ET, Kuo PC, et al. Temporal trends in lung transplant center volume and outcomes in the United States. Transplantation. 2010;89:639-43.

16. Smith PW, Wang H, Parini V, Zolak JS, Shen KR, Daniel TM, et al. Lung transplantation in patients 60 years and older: results, complications, and outcomes. Ann Thorac Surg. 2006;82:1835-41.

17. Tomaszek SC, Fibla JJ, Dierkhising RA, Scott JP, Shen KH, Wigle DA, et al. Outcome of lung transplantation in elderly recipients. Eur J Cardiothorac Surg. 2011;39:726-31.

18. Weiss ES, Merlo CA, Shah AS. Impact of advanced age in lung transplantation: an analysis of United Network for Organ Sharing data. J Am Coll Surg. 2009;208: 400-9. 